A. Pramesh Rao, G. Swarup and Gopal-Krishna, eds.

\title{
The Dual Radio Relics of A3667
}

\author{
M. Johnston-Hollitt, R. W. Clay \\ Department of Physics, University of Adelaide, SA 5005, Australia
}

R. D. Ekers, M. H. Wieringa

ATNF, PO Box 76, Epping NSW 1710, Australia

R. W. Hunstead

School of Physics, University of Sydney, NSW 2006, Australia

\begin{abstract}
.
A3667 is a remarkable cluster of galaxies possessing the two largest and brightest radio relics yet seen. It provides an important opportunity to examine the environments associated with relic production.
\end{abstract}

\section{Introduction}

The ACO cluster A3667 is characterised by a high velocity dispersion, a bimodal galaxy distribution (Proust et al. 1988), and extreme X-ray luminosity, making it one of the brightest, dynamically interesting X-ray clusters in the southern sky. However, the most dramatic feature of this cluster are the two radio relics seen in a symmetrical arrangement bordering the X-ray emission.

Radio data on A3667 were gathered at $0.843,1.4$ and $2.4 \mathrm{GHz}$. The low frequency observations were taken at a resolution of $43^{\prime \prime}$ using the Molonglo Synthesis Telescope (MOST). These MOST images were complemented with higher resolution $\left(13^{\prime \prime}\right) 1.4 \mathrm{GHz}$ data taken with the Australia Telescope Compact Array (ATCA). Data from the ATCA were gathered simultaneously at 1.4 and 2.4 $\mathrm{GHz}$ in four different telescope configurations. Mosaicing was used to cover the large spatial extent of the cluster.

\section{First Results}

Comparison of our radio images and data taken by ROSAT shows there is a connection between the radio and X-ray structure, with the symmetrical arrangement of the relics about the X-ray emitting region clearly evident. Figure 1 shows a $1.4 \mathrm{GHz}$ radio image (greyscale) overlaid with X-ray contours. The alignment of the relics and X-ray emission, combined with the other properties of the cluster, suggests the relics may be the result of propagating shock fronts generated by a cluster merger. Roettiger et al. (1999), have modelled such a merger using N-body magnetohydrodynamics. There is clearly good morpholog- 


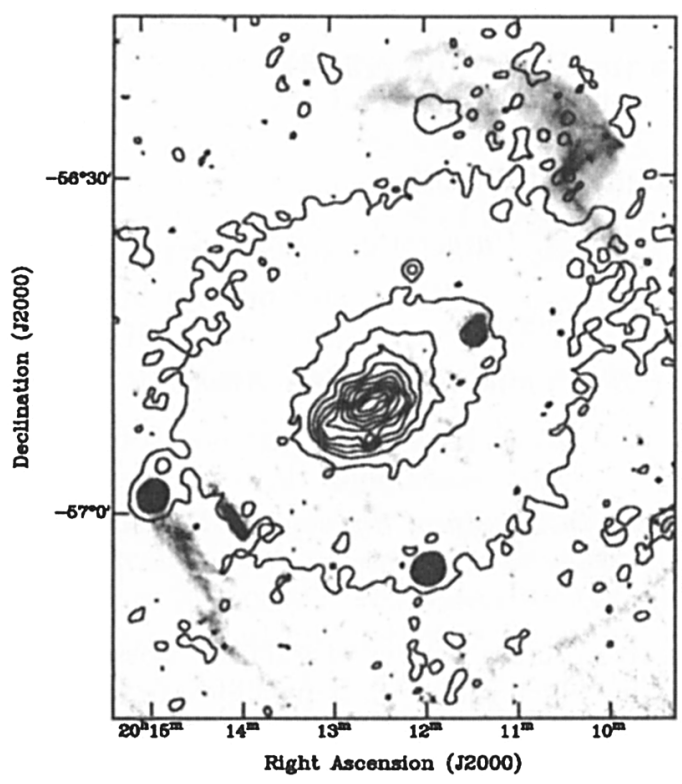

Figure 1. A3667: $1.4 \mathrm{GHz}, 13^{\prime \prime}$, ATCA greyscale image is overlaid with ROSAT PSPC contours. Note the circular features in the image, in particular the one SW corner, are artifacts of the processing and do not correspond to real sources.

ical agreement between their model and our radio data. This is not suprising as a widefield radio image already exists in the literature (Röttgering et al. 1997). However, we also find that our preliminary spectral index data supports the notion that the relics may be slow moving shock fronts (full details will be published elsewhere). The higher resolution images suggest a filamentary structure within the relics which may be associated with the underlying magnetic fields.

We are conducting two further observational campaigns on this cluster. The first, a Faraday rotation measure campaign, aims to use background sources as a probe of the cluster's electron density and magnetic field. The second is a high resolution campaign to probe the observed filamentary structure. The work conducted in these campaigns combined with the present data set will allow us to further probe the environment required to generate relics.

\section{References}

Röttgering, H. J. A., Wieringa, M. H., Hunstead, R. W., \& Ekers, R. D. 1997, MNRAS, 290, 577

Proust D., Mazure A., Sodré, L., Capelato, H \& Lund, G. 1988 A\&AS, 72, 415

Roettiger, K., Burns, K. O. \& Stone, J. M. 1999 ApJ, 518, 603 